九州大学学術情報リポジトリ

Kyushu University Institutional Repository

\title{
Ueber Den Ernährungswert Von Xylan
}

\section{Yamafuji, Kazuo}

Agrikulturchemischen Institut der Kyushu-Universität

Shibatate, Shigero

Agrikulturchemischen Institut der Kyushu-Universität

https://doi.org/10.5109/22639

出版情報 : 九州大学大学院農学研究院紀要. 9 (4)，pp.341-354，1950-11. Kyushu University バージョン：

権利関係 : 
Journal of the Facuity of Agriculture, Kyūshū University, Vol. 9, No. 4

November 30,1950

\title{
UEBER DEN ERNÄHRUNGSWERT VON XYLAN
}

\author{
Kazuo Yamafuji und Shigero Shibatate
}

Wie wir in der vorhergehenden Arbeit festgestellt haben (1), wird Xylan verhältnismässig leicht durch Zusammenwirken von Wasserstoffperoxyd und Eisen zersetzt. Unsere Atmungstheorie, die auf der von uns gefundenen Tatsache der Sauerstoffaktivierung durch Zellgrenzflächen beruht, lehrt, dass das Wasserstoffperoxyd bei den verschiedenen Wegen des terminalen Prozesses der Gewebsatmung gebildet wird. Und vielen Tieren können faserige Stoffe, die meist eine grosse Menge Xylan enthalten, als Nahrungsmittel dienen. Cellulose, die bei den fasrigen Substanzen den Hauptbestandteil darstellt, ist aber gegen biologische Zersetzungen äusserst widerstandsfähig. Folglich ist anzunehmen, dass Xylan, das in der Pflanzenwelt so weitverbreitet vorkommt und so leicht durch biologische Verfahren verzuckert werden kann, ein wichtiger Bestandteil ist, der für pflanzenfressende Tiere Lebensenergie liefert.

Ein typisches Beispiel dafür geben die Lebensvorgänge der Termite, die nur Holz frisst und ein normales Leben führt. Wirklich ergaben unsere Versuche, dass das Verdauungsorgan dieses Insektes eine bedeutend grössere Verzuckerungsfähigkeit für Xylan hat, als für Cellulose. Auch das Rind frisst eine beträchtliche Menge Stroharten. Wir konnten feststellen, dass im Digestionssaft dieses Pflanzenfressers ebenfalls eine sehr starke Xylanase existiert. Andererseits befindet sich im Verdauungskanal der weissen Ratte fast keine oder nur wenige Xylanase. Daher muss bei solchen Tieren Xylose anstatt Xylan gegeben werden. Die Richtigkeit dieser Annahme wurde durch einige Fütterungsversuche bestätigt, bei denen die Ratte mit einem durch Hydrolyse xylanhaltiger Stoffe hergestellten Futter versorgt wurde. 


\section{VERdAUUngSEnzyme DER TERMITE}

Holz, das die einzige Nahrung der Termite ist, enthält über $50 \%$ Cellulose. Die Termite kann sogar von Cellulose allein leben. Cleveland klärte diese merkwürdige Lebensfunktion des genannten Insektes auf; nach ihm wohnen im Verdauungstraktus desselben zahlreiche Protozoen, die die Fähigkeit besitzen, Cellulose zu zerlegen, und weiter nach dem Tod als Stickstoffquelle für den Wirt dienen (2). Nach Trager ist es sehr schwer, aus dem Digestionsapparat der Termite eine starke Cellulase zu isolicren (3). Um die cellulosezersetzende Wirksamkeit möglichst deutlich zu verfolgen, benutzten wir eine Termite, die in einem saftfreien dürren Holz lebt. Für die Prüfung, ob in der Tat das Holz im Termitenkörper zersetzt wird, wurde der Verdauungssaft mit Swezyscher Nährlösung versetzt, auf einem Objektglas fixiert und mit einer Phloroglucinlösung gefärbt. Die dabei verwendete Nährlösung bestand aus $0,3 \mathrm{~g} \mathrm{NaCl}, 0,02 \mathrm{~g} \mathrm{CaCl}_{2}, 0,02 \mathrm{~g} \mathrm{KCl}, 0,01 \mathrm{~g} \mathrm{MgCl}_{2}$, $0,01 \mathrm{~g} \mathrm{NaHCO}, 0,01 \mathrm{~g} \mathrm{NaH}_{2} \mathrm{PO}_{4}, 0,5 \mathrm{~g}$ Blutpulver und $100 \mathrm{ccm}$ Wasser; die Phloroglucinlösung aus $12 \mathrm{~g}$ Phloroglucin, $50 \mathrm{ccr}$ 24\%iger Salzsäure und $50 \mathrm{ccm}$ Wasser. Bei der Mikroskopierung dieses Präparates wurde beobachtet, dass viele Holzstückchen von den Protozoen aufgenommen wurden. Die Holzstücke im Protozoenkörper können durch Rotfärbung von Lignin nachgewiesen werden. Die Frage, ob tatsächlich in der Protozoenzelle eine Cellulosezerlegung vor sich geht, ist noch nicht einwandfrei entschieden.

Zur Bestimmung der Cellulase wurde dem Insekt vorsichtig das Verdauungsorgan entnommen. Der als Substrat zu benutzende Zellstoff ist in Wasser unlöslich, deswegen muss er zur vollstän. digen Berührung mit dem Enzym möglichst fein pulberisiert werden. $\mathrm{Zu}$ diesem $\mathrm{Z}$ wecke wurde reines Filtrierpapier bei etwa $80^{\circ}$ getrocknet, pulverisiert und weiter mit Quarzsand gut zerrieben. Zugleich wurde die cellulosezersetzende Kraft des ganzen Termitenkörpers, einschliesslich des Verdauungsorgans, ermittelt. Bei der Enzymbestimmung wurden beide, der Insektenkörper und das Digestionsorgan, als Gewebebreie verwendet, ohne dass das Enzym isoliert oder gereinigt wurde. Wegen der einfachen Vergleichung mit der Verzuckerungskraft anderer Polyasen und ferner wegen des allgemeinen Mitvorhandenseins der Polyasen mit ent- 
sprechenden Oligasen, wurde die Menge reduzierender Zucker, die nach Bertrand bestimmt wurde, in $\mathrm{mg}$ Glucose ausgedrückt.

Tabelle I. Reaktionsmischung: $0,2 \mathrm{~g}$ Cellulose $+2 \mathrm{~cm} \mathrm{~m} / 15$ Phosphatpuffer vun $\mathrm{pH} 6,8+1 \mathrm{~cm}$ Gewebebrei $+20 \mathrm{ccm}$ Wasser. Reaktionstemperatur: $38^{\circ}$. Reaktionsdauer: 21. Stunden.

\begin{tabular}{c|ccc} 
& Verdauungstinat & Termitenkörper \\
\hline Glucose in $\mathrm{mg} \ldots \ldots \ldots \ldots \ldots \ldots \ldots$ & 2,0 & 11,0 \\
\hline
\end{tabular}

Bei den Versuchen in Tabelle I wurden 100 Individuen be. nutzt; das Gesamtgewicht der Körper war $0,14 \mathrm{~g}$ und das der Verdauungskanäle $0,08 \mathrm{~g}$. Es ist undenkbar, dass in den anderen Geweben ausser dem Digestionsorgan die Cellulase vorkommt. Wahrscheinlich hat sie ziemlich geringe Widerstandskraft gegen äussere Faktoren. $\mathrm{Da}$ das Entnehmen der Verdauungskanäle einige Zeit dauert, besteht die Möglichkeit, dass während dieser Zeit die Aktivität der Cellulase allmählich herabsinkt. In der Tat erfolgt bei längerer Reaktionsdauer keine Zunahme der Zuckermenge. Die Abhängigkeit der Cellulasewirksamkeit von der Wasserstoffionenkonzentration wurde mit dem Brei des ganzen Insektenkörpers ermittelt.

Tabelle II. Reaktionsmischung: $0,3 \mathrm{~g}$ Cellulose $+5 \mathrm{~cm} \mathrm{~m} / 5$ Phosphatpuffer + $1 \mathrm{ccm}$ Gewebebrei $+20 \mathrm{ccm}$ Wasser. Reaktionstemperatur: $37^{\circ}$. Reaktionsdauer: 16 Stunden.

\begin{tabular}{c|c|c|c|c|c|c}
\hline $\mathrm{pH}:$ & 5,6 & 5,9 & 6,5 & 6,8 & 7,2 & 7.7 \\
\hline Glucose in mg..................... & 7,0 & 7,4 & 8,2 & 8,3 & 4,0 & 1,5 \\
\hline
\end{tabular}

Das Reaktionsoptimum der Termitencellulase liegt, wie die Daten in Tabelle zeigen, bei pH etwa 6,6. Dieser Wert entspricht dem pH des Verdauungssaftes der Termite. Die Termitencellu. lase ist in sauer Lösung verhältnismässig beständig, dagegen in alkalischer unbeständig.

Das verzuckernde Vermögen dieses Insektes für die Cellulose, deren Gehalt im Holz am höchsten ist, ist also, wider Erwarten, bedeutend klein. Der Bestandteil, dessen Menge nächst der Cellulose am grössten ist, ist Lignin. Lignin kann aber im allgemeinen in pflanzlichen sowie tierischen Körpern nicht leicht hydrolysiert 
werden, und überdies haben seine Hydrolysenprodukte fast keinen Nahrungswert. Pentosan steht im Prozentsatz unter den Holzbestandteilen an dritter Stelle, und es besteht grösstenteils aus Xylan. Deshalb wurde angenommen, dass das Xylan im Digestionsorgan der Termite zersetzt und von der Wand dessclben absorbiert wird; eine Annahme, die bisher von niemandem geäussert worden ist. Das verwandte Xylan wurde aus Reisștroh dargestellt. Zuerst wurde das Strohpulver mit $2 \%$ igem Ammoniak behandelt und dann mit 7\% iger Natriumhydroxydlösung extrahiert. Der Alkaliextrakt wurde mit Alkohol versetzt, die hier eriaiten Fällung wieder in Alkali gelöst und nochmals mit Alkohol gefällt. Diese Prozeduren wurden noch mehrmals wiederholt. Der letzte Niederschlag wurde schliesslich mit salzsäurehaltigem Alkohol neutralisiert, gewaschen und getrocknet. Als Enzymmaterial wurde ebenfalls der Brei aus 100 Insektenkörpern benutzt.

Tabelle III. Reaktionsmischung: $20 \mathrm{ccm} 3 \%$ ige Xylanlösung $+5 \mathrm{ccm} \mathrm{m} / 15$ Phosphatpuffer von $\mathrm{pH} 6,8+1 \mathrm{ccm}$ Gewebebrei. Reaktionstemperatur : 38. Reaktionsdauer: 20 Stunden.

\begin{tabular}{c|c|c}
\hline & Verdaumgskanal & Termitenküper \\
\hline Xylose in $\mathrm{mg} \ldots \ldots \ldots \ldots \ldots \ldots$ & 6,4 & 18,7 \\
\hline
\end{tabular}

Vergleicht man die Zahlen in Tabelle III mit denen in Tabelle I, so ersieht man, dass im Termitenkörper, wic erwartet, die Xylanaseaktivität sehr viel höher ist, als die Cellulaseaktivität. Es ist dazu zu bemerken, dass die Termitenxylanase, wie Tabelle IV zeigt, bei $\mathrm{pH} 5,3$ am stärksten wirkt und folglich beim $\mathrm{pH}$ Wert wie in den obigen Versuchen ihre Fähigkeit nicht völlig beweist.

Tabelle IV. Reaktionsmischung: $20 \mathrm{ccm} 2 \%$ ige Xylanlösung $+5 \mathrm{ccm} \mathrm{m} / 15$ Phosphatpuffer $+1 \mathrm{ccm}$ Gewebebrei. Reaktionstemperatur: $39^{\circ}$. Reaktionsdauer: 22 Stunden.

\begin{tabular}{|c|c|c|c|c|c|c|c|}
\hline $\mathrm{pH}:$ & 5,0 & 5,3 & 5,6 & 5,9 & 6,5 & 6,8 & 7,4 \\
\hline Xylose in $\mathrm{mg} \ldots .$. & 22,0 & 22,4 & 21,1 & 19,0 & 12,8 & 8,7 & 6,8 \\
\hline
\end{tabular}

Im Verdauungsorgan der Termite leben neben den Protozoen noch zahlreiche Bakterien. Wir haben jedoch keinen Anhaltspunkt, 
ob das xylanverzuckernde Vermögen von den Mikroben herrührt, oder der Termite eigentümlich ist.

Im Holz kommt keine oder nur eine aüsserst geringe Menge Stärke vor. Demnach kann man vermuten, dass die Amylasewirksamkeit der Termite schwach sei. Die Versuche in Tabelle $\mathrm{V}$ lehren aber, dass das Insekt eine bedeutene starke Amylase hat.

Tabelle V. Reaktionsmischnng: $100 \mathrm{ccm} 5 \%$ ige Stärkelösung $+6 \mathrm{ccm} \mathrm{m} / 15$ Phosphatpuffer von pH $6,8+1 \mathrm{ccm}$ Gewebsbrei. Reaktionstemperatur : $39^{\circ}$. Reaktionsdauer: 22 Stunden.

\begin{tabular}{c|c|c}
\hline & Verdautungskanal & Termitenkörper \\
\hline Glucose in $\operatorname{mg} \ldots \ldots \ldots \ldots \ldots \ldots$ & 52,8 & 142,0 \\
\hline
\end{tabular}

Es ist unmöglich, dass die Termite die Hauptquelle für ihre Lebensenergie in den Stärkeabbauprodukten sucht. Vielleicht ist die Verdauungsamylase des Insektes in den Mikrobenkörpern im Verdauungssaft vorhanden und an der Bildung sowie Zersetzung des Glv cogens der Mikroben beteiligt. Der Einfluss der Acidität auf die Termitenamylase wurde durch die Versuche in Tabelle VI aufgeklärt.

Tabelle VI. Reaktionsmischung: $90 \mathrm{ccm} 5 \%$ ige Stärkelösung $+10 \mathrm{ccm} \mathrm{m} / 15$ Phosphatpuffer $+1 \mathrm{ccm}$ Gewebebrei. Reaktionstemperatur: $37^{\circ}$. Reaktionsdauer: 21 Stunden.

\begin{tabular}{c|c|c|c|c|c}
\hline $\mathrm{pH}:$ & 5,9 & 6,5 & 6,8 & 7,2 & 7,7 \\
\hline Glucosc in mg ...................... & 230 & 260 & 240 & 220 & 200 \\
\hline
\end{tabular}

Die optimale Acidität für die Amylasewirkung stimmt mit derjenigen für die Cellulasewirkung überein. Das amylolytische Ferment kommt, von der Cellulase oder Xylanase abweichend, wahrscheinlich auch in verschieden Geweben der Termite vor und nimmt am Glycogenstoffwechsel im Ameisenkörper Anteil.

Um noch eingehendere Kenntnisse von den Eigentümlichkeiten der Termitenpolyasen zu gewinnen, haben wir viele Versuche zur Isolierung der Protozoen aus dem Verdauungsorgan gemacht. Zum Beispiel wurden verschiedene Nährlösungen, wie Strobextrakt, Heuextrakt und Salzgemische, unter wechselnden Konzentrationen sowie pH-Werten verwendet. Die Protozoen stellen selbst unter 
den günstigsten Bedingungen innerhalb einer Stunde ihre Bewegung ein und sterben innerhalb einiger Stunden. Wir konnten also keine Reinkultur der Termitenprotozoen erhalten.

\section{VerdauUngsenzyme DES Rindes}

Das Reisstroh bildet in Japan das wichtigste Futter für die Haustiere. Der erste und grösste Magen des Rindes, der Pansen, ist immer mit Reisstroh angefüllt. In Pansen müssen daher die Strohbestandteile zersetzt und die Zersetzungsprodukte von der Wand des Verdauungsorgans aus absorbiert werden. Substanz, die im Stroh am reichsten vorbanden ist, ist ebenfalls Zellstoff. Deswegen wurde zuerst die Pansencellulase untersucht. Der Pansensaft ist sehr zäh und nicht filtrierbar. Nach der Entfernung fester Körper wurde der Pansensaft unfiltriert als Enzymlösung benutzt.

Tabelle VII. Reaktionsmischung: $0,2 \mathrm{~g}$ Cellulose $+5 \mathrm{~cm} \mathrm{~m} / 15$ Phosphatpuffer von $\mathrm{pH} 7,7+5 \mathrm{ccm}$ Pansensaft $+20 \mathrm{ccm}$ Wasser. Reaktionstemperatur: $40^{\circ}$.

\begin{tabular}{c|c|c}
\hline Reaktionsdauer in Std. : & 24 & 110 \\
\hline Glucose in mg .............. & 2,5 & 9,0 \\
\hline
\end{tabular}

Bei den Versuchen in Tabelle VII wurde die Wasserstoffionenkonzentration der Reaktionsmischung auf diejenige des Pansensaftes eingestellt. Aus den erhaltenen Dates geht hervor, dass die cellulosezersetzende Fähigkeit des ersten Magens des Rindes äusserst niedrig ist. Es besteht also die Möglichkeit, dass sich im Stroh irgendeine Substanz befindet, die vom Rind besscr als Cellulose ausgenulzt wird. Das Reisstroh enthält Xylan zu etwa 25\%. Auf Grund der Erfahrungen an Termite nahmen wir an, dass im Rindermagen ein recht wirksames xylanspaltendes Enzym vorkommt und die entstandene Xyiose zur Wärmequelie für das Rind dient. Demnach wurde hier die Xylanasewirksamkeit des Pansensaftes geprüft.

Die Xylanaseaktivität des Pansens ist, wie erwartet, beträchtlich hoch. Tabelle VIII zeigt auch, dass die Wirkung der Pansenxylanase bei $\mathrm{pH} 6,2 \mathrm{am}$ stärksten ist. Andere Zusatzversuche lehrten ferner, dass das Temperaturoptimum dieses Enzyms bei etwa $40^{\circ}$ liegt. Um die Frage zu lösen, ob die ermittelte Xylanase 
ein eigentliches Gewebsferment des Rindes ist, haben wir dann ein Trockenpräparat der Pansenwand dargestellt. Die Pansenwand wurde zerrieben, mit Aceton behandelt und hierauf im Exsiccator über Schwefelsäure getrocknet. Dieses Präparat besass kein xylanverzuckerndes Vermögen. Im Pansensaft wohnnen, wie bei der Termite, verschiedenartige Mikroorganismen, insbesondere eine grosse Anzahl Bakterien. Daraus wird der Schluss gezogen, dass die Xylanasewirkung nicht aus dem Pansengewebe, sondern aus den Mikroben im Pansensaft stammt.

Tabelle VII. Reaktionsmischung: $0,3 \mathrm{~g}$ Xylan $+10 \mathrm{ccm} \mathrm{m} / 15$ Phosphatpuffer. $+1 \mathrm{ccm}$ Pansensaft $+20 \mathrm{ccm}$ Wasser. Reaktionstemperatur: $40^{\circ}$. Reaktionsdauer: 23 Stunden.

\begin{tabular}{cc|c|c|c|c|c|c|c|c}
\hline $\mathrm{pH}:$ & & 5,3 & 5,9 & 6,2 & 6,4 & 6,8 & 7,4 & 7,7 & 8,0 \\
\hline Xylose in $\mathrm{mg}$ & $\ldots \ldots \ldots$ & 25,4 & 26,8 & 31,1 & 29,3 & 27,5 & 21,5 & 21,3 & 20,4 \\
\hline
\end{tabular}

Dem Rind wird täglich ausser den Stroharten noch eine ziemliche Menge Getreiden gegeben, deren Hauptbestandteil Stärke ist. Deshalb muss die Amylase ein wichtiges Verdauungsenzym dieses Tieres sein. Die Versuche ergaben in der Tat, dass ein starkes amylolytisches Ferment sowohl im Pansensaft als auch in der Pansenwand vorhanden ist Tabelle IX).

- Tabelle IX. Reaktionsmischung: $40 \mathrm{ccm} 5 \%$ ige Stärkelösung $+5 \mathrm{ccm} \mathrm{m} / 15$

1hosphatpuffer von $\mathrm{pH} 7,7+1 \mathrm{ccm}$ Pansensaft oder $2 \mathrm{~g}$ Pansenwand. Reaktionstemperatur: $38^{\circ}$. Reaktionsdauer: 24 Stunden.

\begin{tabular}{|c|c|c|}
\hline & Pansensaft & Pansenwand \\
\hline Glucose in mg ......................... & 54,5 & 58,6 \\
\hline
\end{tabular}

Under den Polyasen des Rinderpansens hat die Amylase die grösste Wirksamkeit. Die Amylase befindet sich in den äusseren sowie inneren Teilen der Pansenwand und kann aus der Wand mit Wasser extrahiert werden. Das optimale $\mathrm{pH}$ dieser Amylasereaktion liegt, dem der Xylanasewirkung entsprechend, bei 6,2.

Der Rinderpansen muss selbstverständlich auch eine eiweisszerlegende Fähigkeit besitzen. Die Protease wurde hier nach Willstätter bestimmt. 
Tabelle X. Reaktionsmischung: 40 ccm $6 \%$ ige Caseinlösung $+5 \mathrm{ccm} \mathrm{m} / 15$ Phosphatpuffer von $\mathrm{pH} 7,7+5 \mathrm{ccm}$ Pansensaft. Reaktionstemperatur : $40^{\circ}$. Reaktionsdauer: 24 Stunden.

\begin{tabular}{c|c|c}
\hline & In 50\%igem Alkohol & In 95\% igem Alkohol \\
\hline Aciditatszunahme in ccm n/5 KOH... & 5,27 & 6,40 \\
\hline
\end{tabular}

Aus Tabelle X geht hervor, dass im Pansen, obgleich er eine Art Magen ist, eine ziemlich starke Protease vorkommt.

\section{FÜtTERUNGSVERSUCHE MIT DER RATTE}

Die obigen Untersuchungen deuten also an, dass für Tiere, die gewöhnlich stärkearme Pflanzenstoffe als Futter aufnehmen, das Xylan die beste Energiequelle darstellt. Infolgedessen wird angenommen, dass auch für Tierc, dic verschiedene Stoffe fressen, das Xylan als Nährmittel dienen kann, wenn es vorher zersetzt wurde. 1st das der Fall, so sind die Versuche, aus Stroharten, Hölzern und anderem in einfacher Weise irgendein Nahrungsmittel oder Futter zu bereiten, sehr hoffnungsvoll. Denn Xylan, dessen Gehalt in derartigen faserigen Stoffen ziemlich hoch ist, ist bedeutend leichter zersetzbar als Cellulose. Ueber den Wert der Xylose als Wärmequelle im tierischen Körper begegnen wir in der Literatur sich widersprechenden Angaben. So gaben Cremer (4) und Thomas (5) an, dass durch Xylosefütterung bei Kaninchen sowie bei Hühnern eine Bildung von Glycogen in der Leber bewirkt wird. Dagegen erfolgt nach Frenzel (6) bei der Xyloseversorgung keine Glycogenbildung im Kaninchenkörper. Neuerdings teilte Iwata (7) in einer Reihe von Arbeiten mit, dass als Folge der Pentosanaufnahme durch Pflanzenfresser in den Muskeln oder in der Leber derselben einigermassen Fett und Glycogen gebildet werden. Es scheint natürlich zu sein, dass in den Körpern der xylanasereichen Tiere die Xylose in Körpersubstanzen umgewandelt wird. Die nächste zu lösende Frage ist die, inwieweit xylanasearme oder xylanasefehlende höhere Tiere und Menschen Xylan sowie Xylose als Quelle ihrer Lebensenergie ausnutzen können. Für diese Untersuchung bildet das Rejsstroh eins der geeignetsten Materialien; denn in Japan wird es jedes Jahr in übermässiger Fülle produziert und dient dort verschiedenen Haustieren als 
Futter. Zuerst muss der Wert des unbehandelten Reistrohs bestimmt werden. $\mathrm{Zu}$ diesem $Z$ wecke wurde unpolierter Reis in verschiedenen Prozentsätzen mit dem Strohpulver gemengt und an Ratten verfüttert.

Tabelle XI. Futtermischung: Reis $45 \sim 95 \%+$ Fischmehl $5 \%+$ Reisstroh $50 \sim 0 \%$.

Futterbestandteil : Rohprotein 8,46 - 13,99\%, Stickstoffreie-Extraktstoffe $60,10 \sim$ $85,79 \%$, Rohfett $2,25 \sim 2,72 \%$, Rohfaser $0,84 \sim 17,30 \%$, Asche $1,23 \sim 9,48 \%$.

\begin{tabular}{c|c|c|c|c|c}
\hline Versuch & $\begin{array}{c}\text { Stroh- } \\
\text { menge } \\
\text { in \% }\end{array}$ & $\begin{array}{c}\text { Anfangskör- } \\
\text { pergewicht } \\
\text { der Ratte in g }\end{array}$ & $\begin{array}{c}\text { Endkörper- } \\
\text { gewicht der } \\
\text { Ratte in g }\end{array}$ & $\begin{array}{c}\text { Köpergewichts. } \\
\text { zunahme der } \\
\text { Ratte in gufgenommene }\end{array}$ & $\begin{array}{c}\text { futtermenge } \\
\text { wahrend ver Ver. } \\
\text { suchsdauer in g }\end{array}$ \\
\hline 0 & 90 & 141 & 51 & 1240 \\
5 & 94 & 14.4 & 50 & 1230 \\
10 & 112 & 163 & 51 & 1215 \\
20 & 102 & 158 & 56 & 1280 \\
0 & 57 & 158 & 101 & 1260 \\
15 & 58 & 161 & 103 & 1250 \\
30 & 60 & 158 & 98 & 1550 \\
50 & 62 & 160 & 98 & 1710 \\
\hline
\end{tabular}

Die Fütterungsexperimente wurden immer vom Standpunkte der chemischen Analyse sowie der Ernährungsmethode aus möglichst in eingehender Weise durchgeführt. Hier werden jedoch nur kurz einige Auszüge aus zahlreichen Daten beschrieben. Auch bei den Versuchen mit dem unbehandelten Stroh wurden nur die wesentlichen Ergebnisse in Tabelle XI zusammengestellt. Das Körpergewicht und die Futtermenge sind die Durchschnittswerte der Weibchen. Aus den erhaltenen Zahlen erkennt man, dass das Reisstroh in diesem Zustand einc ziemlich hohe Ernährungsfähig. keit besitzt. Die Ratte wächst normal heran, selbst wenn die Hälfte der Futtermischung aus dem Strohmchl besteht. 1st der Strohgehalt des Futters unter $20 \%$, so sind Wachstumsgeschwindigkeit des Tieres sowie aufgenommene Futtermenge beinahe gleich wie beim Grundfutter ohne Stroh. Beträgt aber der Strohgehalt über $30 \%$, so frisst das Tier die Futtermischung um etwa den gemischten Prozentsatz des Strohmehls mehr als ungemischtes Grundfutter allein, um mit dem Wachstum des Kontrolltieres Schritt zu halten. Der so hohe Nährwert des Reisstrohs beruht wahrscheinlich nicht nur auf seinem grossen Xylangehalt, sondern 
auch auf seiner krautigen Natur. Da nämlich die Reispflanze eine Art Gras ist, wird es in der Form des ganzen Pffanzenkörpers, abgesehen von der Wurzel, den Haustieren gegeben. Das Strohfutter enthält demnach verschiedene Pflanzenteile, besonders Blätter, und an nahrhaften Bestandteilen, besonders Eiweiss und Vitamine. Die Analyse des Reisstrohs ergab in der Tat: Rohprotein 5,64\%, Stickstoff reie-Extraktstoffe 36,40\%, Rohfett 1,33\%, Rohfaser 37,13\% and Asche 19,50\%. Wie aus unseren Befunden an Verdauungsenzymen der Termite oder des Rindes ersichtlich ist, wird der Ernährungswert des Reisstrohs noch erhöht, wenn das darin befindliche Xylan im voraus verzuckert wurde.

Für das Zweck der Umwandlung der Strohmaterialien in nahrhaftere Futter oder Nahrungsmittel sind kostbare Apparate und Verfahren nicht praktisch. Um möglichst einfache Methoden zu finden, wurden die Versuche unter verschiedenen Bedingungen angestellt. Bei den Methoden, die bisher von vielen Forschern angeführt wurden, wurden faserige Stoffe nach der Behandlung mit Säuren oder Alkalien gewaschen und erhaltene Rückstände zu den Tierexperimenten verwendet. Nach solchen Prozeduren werden aber leicht zersetzbare, folglich auch leicht absorbierbare lösliche Substanzen entfernt. Deshalb haben wir in der verliegenden Arbeit fast immer nach der Zersetzung des Strohs neutralisiert und die wasserlösliche Substanzen enthaltenden Präparate dargestellt. Vorläufige Versuche zeigen, dass der grösste Teil des Xylans im Reisstroh durch verhältnismässig milde chemische Mittel, zum Beispiel durch 4stündiges Erhitzen mit 2\%iger Schwefelsäure im siedenen Wasserbad verzuckert wird. Die Versuche, passende Katalysatoren aufzufinden, die die Xylanverzuckerung $\mathrm{zu}$ beschleunigen vermögen, waren erfolglos. Beispielsweise wird in Gegenwart von Natriumfluorid, das eine Fähigkeit besitzt, die Sauerstoffbrücken im Cellulosemolekül zu spalten (8), die einmal entstandene Xylose weiter zersetzt. Obwohl das Strohxylan durch Säure von höherer Konzentration noch leichter zerlegt wird, ist nach unseren Erfahrungen über 4\% \%ige Schwefelsäure für den industriellen Gebrauch bei der Bereitung der Nahrungsmitteln nicht anwendbar. Denn ein Präparat, welches hergestellt wurde, indem das Strohpulver zuerst mit solcher Säure hydrolysiert, dann mit Calciumhydroxyd neutralisiert und weiterhin der Rückstand zusammen mit der Flüssigkeit getrocknet wird, wirkt auf das 
Wachstum der Ratte besonders dann störend, wenn es in einer Proportion über $30 \%$ mit dem Grundfutter gemischt wird.

Die Strohbestandteile werden auch durch Alkalien zersetzt. Durch alkalische Reagenzien wird aber reduzierender Zucker, der eins der wichtigsten Nahrungsbestandteile ist, gespaltet. In Tabelle XII sind die Resultate der Pentosanzersetzung durch Calcium. hydroxyd im Vergleich mit denjenigen der Säurespaltung des Pentosans wiedergegeben.

Tabeile XII. Reaktionsmischung: $30 \mathrm{~g}$ Reisstroh $+150 \mathrm{ccm}$ $\mathrm{Ca}(\mathrm{OH})_{2}$-Lösung od. $\mathrm{H}_{2} \mathrm{SO}_{4}$.

\begin{tabular}{c|c|c|c|c|c}
\hline Reagens & $\begin{array}{c}\text { Konzentration } \\
\text { des Reagens } \\
\text { in \% }\end{array}$ & $\begin{array}{c}\text { Erhitzungs. } \\
\text { temperatur } \\
\text { in C }\end{array}$ & $\begin{array}{c}\text { Erhitzungs- } \\
\text { dauer in Std. }\end{array}$ & $\begin{array}{c}\text { Pentosan im } \\
\text { Präparat } \\
\text { in \% }\end{array}$ & $\begin{array}{c}\text { Pentose im } \\
\text { Praparat } \\
\text { in \% }\end{array}$ \\
\hline $\begin{array}{c}\text { Ohne } \\
\text { Reagens }\end{array}$ & - & - & - & & \\
$\mathrm{Ca}(\mathrm{OH})_{2}$ & 1 & 100 & 5 & 24,1 & 2,57 \\
$\mathrm{Ca}(\mathrm{OH})_{9}$ & 2 & 100 & 5 & 14,7 & 0,56 \\
$\mathrm{H}_{\mathbf{2}} \mathrm{SO}_{4}$ & 2 & 100 & 4 & 11,6 & 0,52 \\
$\mathrm{H}_{2} \mathrm{SO}_{4}$ & 4 & 100 & 2 & 5,5 & 15,20 \\
\hline
\end{tabular}

Bei der Herstellung der Präparate, die in den obigen Versuchen benutzt wurden, wurde das Reisstroh nach der Hydrolyse durch Calciumhydroxyd oder Schwefelsäure mit Schwefelsäure oder Calciumhydroxyd neutralisiert. Wie aus diesen Daten zu erwarten ist, wird der Nahrungswert des Strohs durch die Kalkzersetzung nur cin wenig erhöht. Die Erhöhung des Nährwertes ist hierbei auf das Weichwerden der Strohfaser durch Alkali zurückzuführen.

Reagenzien, die bei der Herstellung der Strohpräparate verwerden, können dadurch erspart werden, dass man die mittels einer Säure zersetzten Präparate nicht mit Alkalien, sondern mit den mittels eines Alkalis zersetzten Präparaten neutralisiert. Da aber unter der Wirkung des Alkalis eine Zuckerspaltung stattfindet, wird angenommen, dass die in solcher Weise dargestellten Strohpräparate einen niedrigeren Nährwert haben, als die mit einer Säure allein zerlegten Präparate. Die Richtigkeit dieser Annahme wurde ebenfalls durch die Fütterungsversuche an weissen Ratten bewiesen.

Auf dem Stroh leben gewöhnlich Mikroben, die imstande sind, Cellulosemoleküle abzubauen. Die Herstellung eines Komposthau- 
fens aus Stroh beruht auf der Wirkung derartiger Mikroben. Wenn eine wässerige Suspension des Strohpulvers bei Zimmertemperatur stehen gelassen wird, so erfolgt in der Tat eine Abnahme des Cellulosegehaltes. 1st aber die Einwirkungsdauer der Mikroben kürzer als eine Woche, so ist der Zersetzungsgrad der Cellulose nur gering. Gleichzeitig wird auch eine Spaltung des Pentosans beobachtet; die Pentosanverminderung ist gleichfalls klein. Ausserdem erfahren die gebildeten Monosen durch die Mikrobenenzyme eine weitere Zerlegung und die Menge des reduzierenden Zuckers in den dargestellten Strohpräparaten wird noch geringer als im Ausgangsmaterial. Aus diesen Befunden ist anzunehmen, dass der Futterwert des Reisstrohs durch solche Veränderungen nicht so gesteigert wird. In Wirklichkeit zeigten die Tierexperimente, dass die Erhöhung der Ernährungsfähigkeit des Strohs durch die Wirkụng derartiger Mikroorganismen gering ist.

Die Natur, das Molekulargewicht und folglich auch die Zersetzbarkeit der Cellulose, sind bis zu einem gewissen Grade von dem Ursprung abhängig. Die Versuche lehrten, dass die Cellulose des Reisstrohs gegenüber noch konzentrierterer Säuren ziemlich beständig ist. Die Glucosebildung aus der Cellulose ist gering, selbst wenn zum Beispiel das Strohlmehl 8 Tage bei $40^{\circ}$ in einer 20 bis $30 \%$ igen Schwefelsäure liegt. Obwohl dabei eine ziemliche Menge reduzierender Zucker gebildet wird, besteht der grösste Teil derselben aus der Pentose. Die Pentosanzersetzung geht also unter solchen Versuchsbedingungen bedeutend stark vor sich. Wird das Reisstroh eine Stunde mit 20-30\% iger Schwefelsäure auf etwa $90^{\circ}$ erhitzt, so wird das Pentosan fast vollständig zerlegt, während sich die Monosenmenge wegen der zu kräftigen Säure. wirkung vermindert. Wir haben dann unter der Anwendung der Schwefelsäure in gleicher Verdünnung einige Strohpräparate hergestellt. Das Reisstroh wurde 2 Tage bei $40^{\circ}$ oder 1 Stunde bei $90^{\circ}$ mit $30 \%$ iger Schwefelsäure zersetzt, filtriert und das Filtrat mit Calciumhydroxyd nentralisiert. Die Calciumsulfat abfiltrierte Lösung wurde mit dem Strohrückstand gemischt und getrocknet. Die erhaltenen Präparate wurden im Verhältnis von $20 \%$ mit einem Grundfutter gemengt und den Ratten gegeben. Dabei wurde gefunden, dass die Tiere bei der Versorgung mit solchen Futtermischungen schneller heranwachsen, als wenn sie Mischungen mit unbehandeltem Stroh fressen. 
Aus den oben beschriebenen Tatsachen wird der Schluss gezogen, das Xylan im Reisstroh nach der Hydrolyse als Wärmequelle im Rattenkörper ausgenutzt werden kann. Wenn dies der Fall ist, so müssen Präparate, die Xylanabbauprodukte enthalten, einen höheren Ernährungswert haben, als Präparate, von denen derartige Abbauprodukte befreitet wurden. Das Strohpulver wurde 6 Stunden mit 1\% iger Schwefelsäure auf $85^{\circ}$ erwärmt, ein Teil davon mit Calciumhydroxyd neutralisiert und ein anderer ohne Neutralsierung filtriert. Bei der Herstellung des ersteren Präparats wurde der Strohrückstand zusammen mit der Lösung und bei der des zweiten der Rückstand allein getrocknet. Die Zusammensetzungen der beiden Strohpräparate sind in Tabelle XIII widergegeben.

Tabelle XIII. Zahlen: \% des einzelnen Bestandteils in der Trockensubstanz des Präparats.

\begin{tabular}{|c|c|c|c|c|c|c|c|}
\hline & $\begin{array}{l}\text { Roh- } \\
\text { protein }\end{array}$ & $\begin{array}{l}\text { Stick- } \\
\text { stoffreie } \\
\text { Extrakt- } \\
\text { stoffe }\end{array}$ & Rohfett & Rohfaser & Asche & Pentosan & $\begin{array}{l}\text { Reduzie- } \\
\text { render } \\
\text { Zucker }\end{array}$ \\
\hline $\begin{array}{c}\text { Neutralisiertes } \\
\text { Präparat }\end{array}$ & 3,97 & 25,46 & 0,67 & 18,57 & 31,12 & 16,26 & 4,13 \\
\hline $\begin{array}{l}\text { Filtriertes } \\
\text { Präparat }\end{array}$ & 4,39 & 37,35 & 0.73 & 24,90 & $16,0.3$ & 16,04 & 0,56 \\
\hline
\end{tabular}

Beim filtrierten Präparat ist, wi trwartet, die aus Strohxylan gebildete Xylose beseitigt worden. Die Ernährungsexperimente wurden mit einer Futtermischung von $80 \%$ Grundfutter und 20\% Strohpräparaten ausgeführt (Tabelle XIV).

Tabelle XIV. Futterbestandteil: Angegeben getrennt; neutralisiertes Präparat, filtriertes Präparat; Rohprotein 21,52\%, 21,64\%: Stickstoff reie-Extraktstoffe $55,44 \%, 57,82 \%$; Rohfett $2,78 \%$. $2,79 \%$; Rohfaser $6,25 \%, 7,52 \%$; Asche 9,97\%, 6,95\%.

\begin{tabular}{c|c|c|c|c}
\hline & $\begin{array}{c}\text { Anfangskorper- } \\
\text { gewicht der } \\
\text { Ratte in g }\end{array}$ & $\begin{array}{c}\text { Endkörperge- } \\
\text { wicht der } \\
\text { ratte in g }\end{array}$ & $\begin{array}{c}\text { Körpergewichts- } \\
\text { zunahme der } \\
\text { Ratte in g }\end{array}$ & $\begin{array}{c}\text { Aufgenommene } \\
\text { Futtermenge } \\
\text { wahrend der } \\
\text { Versuchdauer } \\
\text { in g }\end{array}$ \\
\hline $\begin{array}{c}\text { Neutralisations- } \\
\text { gruppe....... }\end{array}$ & 74 & 160 & 86 & 752 \\
$\begin{array}{c}\text { Filtrations. } \\
\text { gruppe ......... }\end{array}$ & 74 & 154 & 80 & 744 \\
\hline
\end{tabular}


Die angeführtdn Resultate betreffen nur die Männchen. Die Körpergewichtszunahme wird desto langsamer, je mehr die Ratte sich der Pubertät nähert und die Ergebnisse wurden allmählich undeutlicher. Um den Einfluss der Stropräparate auf das Rattenwachstum deutlich zu zeigen, wurden in dieser Versuchsreihe nur die Daten in der Zeit angegeben, in der die Tiere noch im kräftigen Wachstum stehen. Die Mengen der Nahrungsstoffe sowie der Energie, die für die Körpergewichtszunahme von $100 \mathrm{~g}$ notwendig sind, lehren Tabelle XV.

Tabelle XV. Versuchtier: Ratten mannchen.

\begin{tabular}{|c|c|c|c|c|c|c|}
\hline & $\begin{array}{c}\text { Nahrung } \\
\text { in } g\end{array}$ & $\begin{array}{l}\text { Rohpro- } \\
\text { tein in } g\end{array}$ & $\begin{array}{l}\text { Stickstoffreie } \\
\text { Extraktstoffe } \\
\text { in } \mathrm{g}\end{array}$ & $\begin{array}{l}\text { Rohfett } \\
\text { in } g\end{array}$ & $\begin{array}{l}\text { Energie } \\
\text { in kcal }\end{array}$ & $\begin{array}{l}\text { Energie } \\
\text { Verhaltnis }\end{array}$ \\
\hline $\begin{array}{l}\text { Neutralisations- } \\
\text { gruppe } \ldots \ldots \ldots \ldots\end{array}$ & 874 & 188 & 484 & 23 & 2875 & 100 \\
\hline $\begin{array}{l}\text { Filtrations- } \\
\text { gruppe } . . . . . .\end{array}$ & 930 & 200 & 633 & 25 & 3557 & 122 \\
\hline
\end{tabular}

Das Reisstroh wurde ferner mit Calciumhydroxyd in ähnlicher Weise zersetzt und zwei ähnliche Präparate hergestellt. Da aber unter der Einwirkung des Alkalis keine Anhäufung der Monosen erfolgt, ist hierbei der Erhöhungsgrad des Nährwertes durch die Neutralisation kleiner, als bei der Säurezersetzung.

Durch die oben erwähnten Versuche konnte der Ernährungs. wert des natürlichen, in Pflanzen befindlichen Xylans für verschiedene Tiere nachgeweiesen werden. Die Ernährungsversuche mit dem rein isolierten Xylan sind im Gange.

Die Arbeit wurde durch ein Stipendium des Unterrichtsministeriums unterstützt.

(Aus dem Agrikuiturchemischen Institut der Kyushu-Universität in Fukuoka, Japan).

\section{LITERATUR}

1) Yamafuji u. Urakami, J. Fac. Agr., Kyushu Univ. 9, 333, (1950)-_-2) Cleveland, Am. Jl. Hyg. 3, 444 (1923); Quart. Rev. Biol. 1, 51 (1923)._-3) Tragcr, Biochem. Jl. 26, 1762 (1932).—4) Cremer, Zs. Biol. 29, 484 (1892).-5) Thomas, Gradinescu u. Imas, Chem. Zentraibl. 1929, 1, 2897.-6) Frenzel, Pflüger's Arch. 56, 273 (1894). --7) Iwata, Jl. agricult. chem. Soc. Japan 6, 804 (1930); 8, 1175, 1252 (1932).8) Fredenhagen u. Helferich, Zs. angew. Chem. 46, 113 (1933). 